\title{
A PROSTHESIS FOR BELOW-KNEE AMPUTEES
}

\author{
By Roy E. Pfaltzgraff, m.D.
}

Garkida Leprosarium, Garkida via Yola, Nigeria.

\section{Introduction}

Recently there has been considerable material published concerning the problem of the care of the foot in leprosy. These contributions have added materially to our knowledge of the mechanism of foot ulceration; of how to prevent it, and how to treat it when it does occur. However, the problem of ulceration in the anaesthetic foot is not completely solved, and it will be many years before we have seen the last refractory foot ulceration under control. All will agree that at the present time there is a group of leprosy patients with such severe foot abnormalities that no presently known method of management will bring about satisfactory rehabilitation.

Our own management of foot ulceration includes the standard methods of treatment including surgery (carefully conserving any functional tissues), bed rest, walking casts, and a rigid soled prosthetic shoe. Only after several years of persistent treatment without effecting a cure have we resorted to amputation.

In the past two years we have had ten cases where a below-knee prosthesis was indicated. These cases can be divided into three categories. (1) Those who had had previous amputation, of which there were three cases. (2) Those with malignant degeneration in a long standing ulcer. There were four of these. (3) The third group was made up of three patients with ulcers which refused to heal or to stay healed for any length of time over a trial period of at least four years. Two of these were constantly in hospital with ulcerations of the foot.

The results in these ten people are as follows: Eight are fully rehabilitated. One died of metastasis from the original malignancy, and one has not yet been fitted. The only apparent failure is the patient who died, and she too was ambulatory with this type of prosthesis prior to her final illness.

We have had no training in the manufacture of prostheses, and thus much study, thought and experimentation were involved in the preliminary stages of the development of this prosthesis. There were also no preconceived ideas as to how the work should proceed, and thus some revolutionary principles in prosthesis manufacture have been evolved.

In our situation there were certain goals to be met in this project which we list here:

1. The prosthesis must be practical and functional. It must be strong, sturdy, durable and lightweight.

2. It must make the patient ambulatory without crutches so that he or she can do farmwork or housekeeping. 
3. It must be manufactured cheaply.

4. It must be simply constructed so that the manufacture can be carried out by a person who has not had technical training in prosthetics.

5. The process of construction must not be time consuming.

\section{Materials Required}

1. Woven cotton stockinette of 3 in. and 4 in. widths.

2. One-eighth inch thick foam rubber. 'Rubazote' made by Dunlop.

3. One-quarter inch thick foam rubber. 'Sorbo' rubber, B.F.S. Grade, made by Dunlop.

4. Rubber Cement. Commercial impact adhesives are preferable, such as 'Evostik' Impact Adhesive 528, manufactured by Evode Ltd., Stafford, England.

5. Epoxy Resin of a type suitable for cloth impregnation and lamination technique, such as 'Orthobond A \& E', Vernon-Benshoff Co., Pittsburg 30, Pa., or 'Epicote $815^{\prime}$ ' with Curing Agent, Shell Co. Ltd.

6. Lightweight wood, such as kapok or obeche.

7. Other supplies such as cardboard, cord, tyre rubber, etc., which are available anywhere.

8. Simple tools of a type available anywhere.

\section{Technique of Construction}

1. The first requirement is that the stump be well healed and shrunk by at least a month to six weeks of constant application of an elastic bandage.

2. The prosthesis is manufactured directly on the stump itself. Note that radical departures from customary prosthetic construction are printed in italics.

3. The stump is examined for any pressure points, and if there are any sites which may possibly break down due to pressure, a small pad of cotton is shaped to cover the area and is glued in place directly over the danger site with rubber cement. The most common danger point is the distal portion of the anterior tibial crest. The cotton pad makes a depression in the final prosthesis so that there is less pressure on the tissues in that area.

4. The stump is liberally powdered so that difficulty will not be encountered on removing the prosthesis.

5. A piece of stockinette which will fit the stump snugly is cut of proper length to cover the stump, and with adequate extra length to eventually form the outside layer (covering) of the completed prosthesis (A in Fig. 1). That is, it must be the length of the stump to the apex of the patella plus the distance from the apex of the patella to the floor; remembering that as the stockinette is stretched laterally 
its length is diminished. The end of the stockinette is then sewn shut in a curve which will make a snug fit over the end of the stump.

6. The stockinette is then slipped on the stump with the extra length pushed up above the knee temporarily. See Fig. 1.

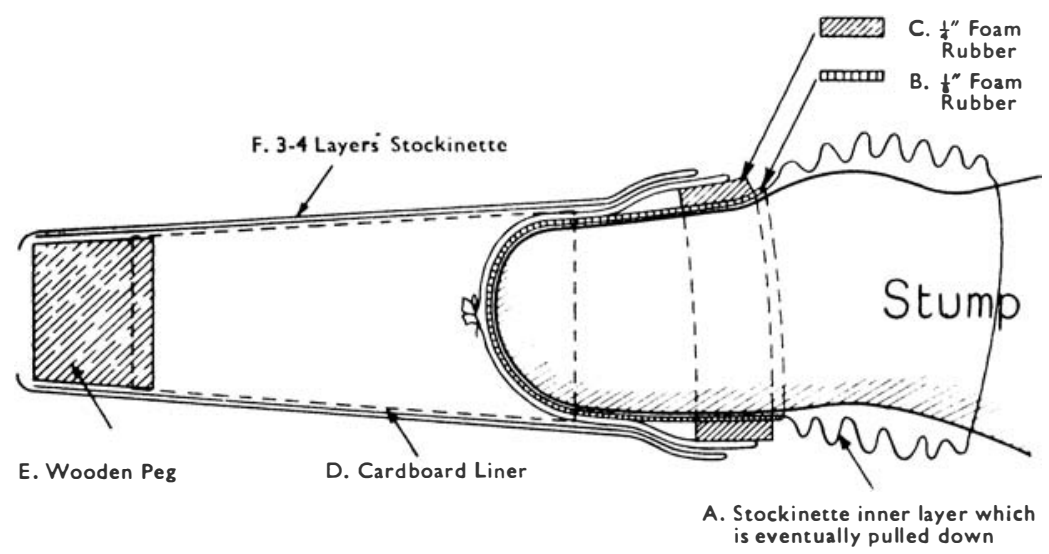

Fig. 1 Diagrammatic Cross-section of completed Prosthesis showing various layers.

7. The stockinette beyond the seam over the end of the stump is glued and turned back on itself so as not to produce an elevation on the inside of the prosthesis.

8. A layer of $\frac{1}{8}$ in. foam rubber is then measured, cut and glued to cover the entire stump from the apex of the patella downward (Fig. 1-B). It must be carefully fitted over the end of the stump so as not to cause any irregularities. This is accomplished by allowing it to extend about $1 \frac{1}{2}$ in. below the end of the stump, and then with the fingers carefully pushing the overlap in, in four quadrants so that they adhere to themselves, leaving four tags which are then cut off flush with scissors. See Fig. 2.

9. A strip of $\frac{1}{4} \mathrm{in}$. Sorbo foam rubber is then cut $3 \mathrm{in}$. wide and long enough to go around the stump just below the knee (C in Fig. 1). Leave about $\frac{1}{4}$ in. of the $\frac{1}{8}$-in. rubber extending above the heavier cuff. The $\frac{1}{4}$ in. cuff is then glued into place under slight tension; care being taken that the underlaying layers of foam rubber and stockinette do not wrinkle.

Note: This is applied with tension in order that on insertion of the stump the prosthesis will grasp it just below the knee, and prevent the prosthesis from slipping. We have not been successful in all instances in getting this sufficiently tight, but where it has been adequately so it has simplified the procedure and is less cumbersome than a retaining strap which is needed if this constricting band is not tight enough. 


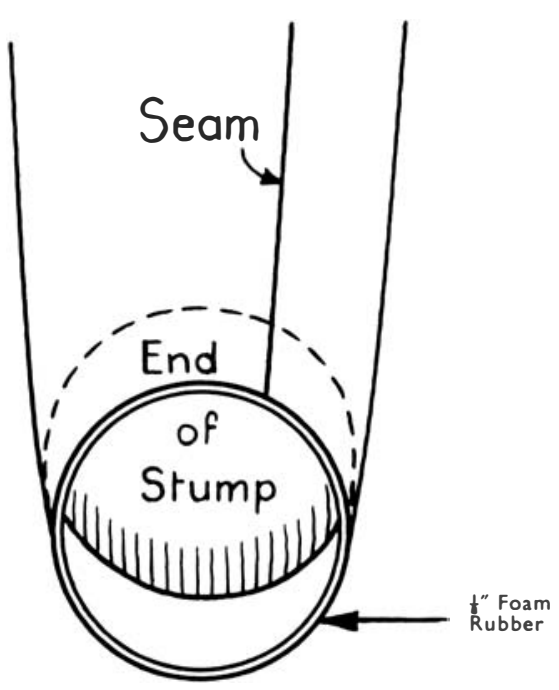

A. Foam Rubber Cylinder

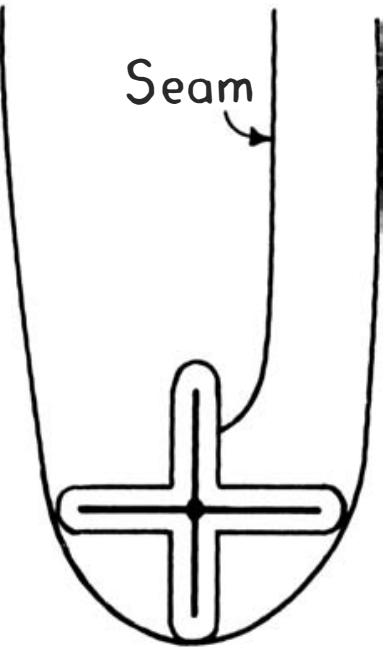

B. Closure in 4 Quadrants

Fig. 2. Closure of Foam Rubber laver over end of Stump.

10. The second innovation - The supporting portion of the prosthesis is a plastic resin which is applied directly over the ahove preparation while on the limb.

11. A second layer of stockinette is then cut a bit more than twice the length of the stump. The end is again sewn shut, and it is slipped up over the stump with the second thickness, which will be turned down later, pushed up on the knee.

12. The plastic which is to be used is then prepared for application to the stockinette according to the manufacturer's directions. The impregnation should be higher on the limb anteriorly so that it extends up to the apex of the patella; but posteriorly it should be lower so as to allow for free movement of the hamstring tendons during flexion of the knee.

The plastic must be applied adequately to thoroughly impregnate the stockinette, which will take from 6 to 8 tablespoonsful of the mixture. It is best applied with a spoon. After the first layer is impregnated the short piece of stockinette at the top is turned down on itself and tied shut with a bit of cord over the end of the stump, and this layer also impregnated thoroughly.

13. After the impregnation an ordinary muslin bandage is used to snugly wrap the stump completely. This will take up any extra plastic which may be squeezed out of the stockinette.

N.B. WARNING! Do not use too much plastic at this stage thinking to make a sturdier prosthesis, as the chemical reaction which occurs on the solidification of the plastic produces heat, and it is possible to produce a burn. If the layers are not thicker than recommended above, the heat will be dissipated innocuously. If there is 
concern that this will not be strong enough, a second layer of plastic could be added after removal from the stump, although we have not found this necessary.

14. The limb should then be supported above the knee so that no pressure is put on the prosthesis so as to distort it during the hardening process. It is then left unmolested for at least two hours; the time depending upon the setting time of the plastic used.

15. When the plastic has become stony hard the entire prosthetic shell is removed from the limb. The anterior border is marked to correspond to the midline of the patella.

16. Any of the muslin bandage which has not taken up plastic and thus been incorporated into it, is now pulled or trimmed off.

17. Measure the length of the other leg to determine the length which will need to be added to the prosthesis to make it equal the other limb.

18. Some sort of jig is now needed to hold the inner shell of the prosthesis (completed in 17 above), and the wooden peg which is used to make the distal end of the prosthesis. This peg is made of lightweight wood and shaped as a truncated cone. The proximal end should be about 3 in. in diameter, and tapered slightly distally. We have made the necessary jig simply from a few blocks of wood, and straps to hold the prosthesis pieces made from inner-tube rubber. (A lathe would make an excellent jig.)

19. The inner shell, and the wooden end piece are now lined up in the jig, and held firmly in the position which is necessary in the final prosthesis. This should be only slightly longer than will be necessary in the final fitted prosthesis. See Fig. 3.

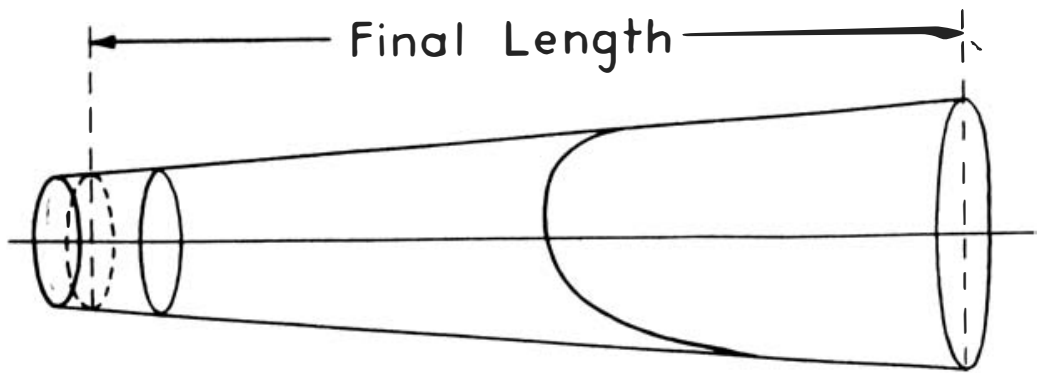

FIG. 3. Diagram showing line-up of inner shell and end piece.

20. With rubber contact cement a piece of heavy paper or corrugated cardboard is now glued as a cylinder (conical) connecting these two pieces.

21. This paper is then impregnated with plastic while held in the jig. When it has set, depressions or irregularities can be filled in with some cheap mixture to make the prosthesis cylindrical and symmetrical. (We use sawdust mixed with prevulcanized latex for this). This must be left set to harden and dry before proceeding. 
22. Now three or four layers of stockinette are used to cover the entire prosthesis, and are impregnated carefully with plastic layer by layer, remembering to carry the plastic high on the prosthesis anteriorly, and lower posteriorly to allow for the hamstring tendons.

23. Finally the original long length of stockinette which made up the innermost layer of the stump socket is pulled down the outside over all and tied shut over the wooden end plug, and also impregnated with plastic.

24. The cord used to tie this shut is also convenient for hanging up the prosthesis in a place where it will not be disturbed while setting.

25. A careful remeasurement is now made of the required length of the prosthesis, and the end is cut off with a carpenter's saw.

26. A piece of rubber tyre is then glued and secured with a few $\mathrm{I}$ in. nails to the distal end.

The prosthesis is now ready for use. The majority of our patients have been able to use the prosthesis without further alteration. In some cases, however, the elastic cuff below the knee was not tight enough and it was necessary to add an above-knee supporting strap.

\section{Strap for supporting Below-Knee Prosthesis}

This strap is a very simple and unique method of supporting a below-knee prosthesis.

\section{Procedure for making and fitting this Strap}

1. The strap is made from rubber sheeting which is cut from automobile tyre inner tubing, which should be cut about 2 in. wide. A flat strap can be cut from an inner tube by cutting the entire tube round and round on a spiral, taking care that at the centre of the tube the strip is of the required width ( $2 \mathrm{in}$.). The tube is cut as in the diagram Fig. 4, and the strip obtained looks as shown in Fig. 5, and merely needs trimming of the edges to get a uniform, strong, elastic strap which lies perfectly flat.

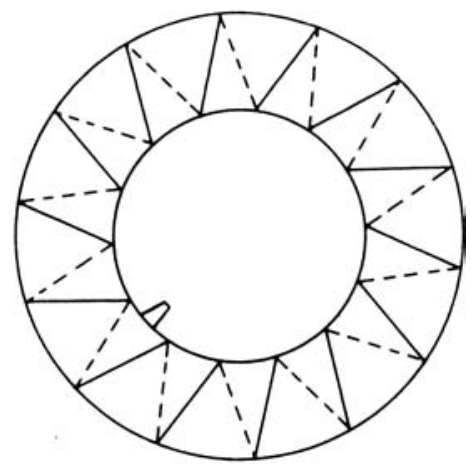

FIG. 4. Manner of cutting Inner Tube.

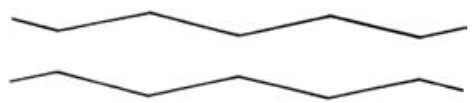

FIG. 5. Strap obtained from Tube. 
2. The strap is attached to the prosthesis by either two or four $\frac{1}{2}$ in. sheet metal screws inserted on the popliteal side as in Fig. 6.

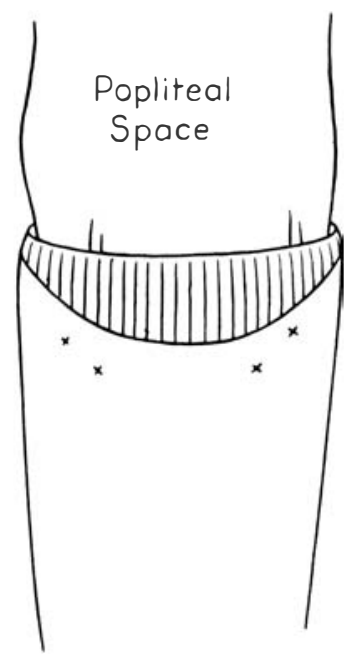

FIG. 6. Site of fixing Supporting Strap.

If it is found that the plastic does not extend near enough to the knee or is not thick enough in this area a bit can be added by surrounding the prosthesis near the top with a cuff of plastic impregnated stockinette.

3. The strap is then punched to fit over the one or two medial screws. It runs from there across the popliteal space posteriorly, laterally and superiorly and crosses the anterior of the leg superior to the patella. Here it turns inferiorly and medially and crosses itself on the posterior of the knee and is attached to the one or two lateral screws. It is well to rub the heads of the screws with chalk, then pull the rubber strap with some tension across them, thus marking the site for the holes with the chalk covered screw head. The chalkmarks thus show the propersites for punching the holes in the strap. See Fig. 7.

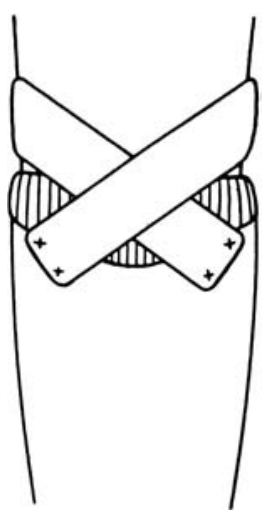

Posterior

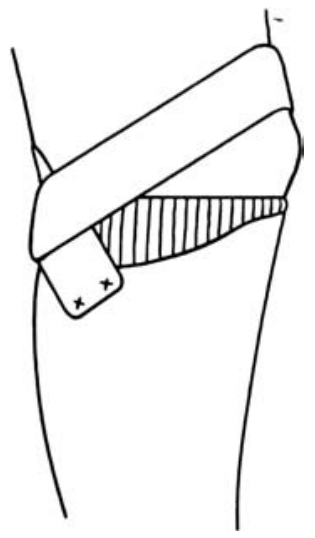

Lateral

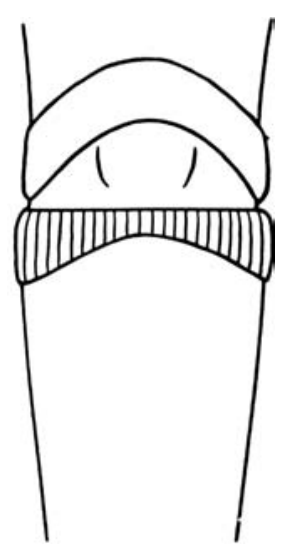

Anterior

FIG. 7. Diagram depicting the manner of application of Strap. 
This prosthesis fits very satisfactorily, and most patients have had no trouble walking with it immediately. At first its use must be graduated, and the enthusiastic patient must be curbed or ulceration may occur before the stump is sufficiently toughened to withstand weight-bearing. 